\title{
Circulation on the Continental Shelf
}

\author{
(coastal circulation/salinity/North America/shelf)
}

\author{
HENRY STOMMEL AND ANTS LEETMAA
}

Massachusetts Institute of Technology, Cambridge, Mass. 02139

Contributed by Henry Stommel, September 13, 1972

\begin{abstract}
A simple analysis is presented of a hypothetical shelf-circulation that could apply to the eastcoast continental shelf of North America under winter conditions.
\end{abstract}

The physical processes that govern the circulation of the waters overlying continental shelves are likely to be different for different shelves and are likely to vary with season. Observational material is extremely limited; as a result, it is not possible to identify with certainty the mechanism operating on a particular shelf. In such a situation it is of some interest to advance hypothetical models in order to discover how well they can account for the fragmentary information that is presently available, with an eye toward finding indications of types of new data particularly desirable, and hints as to directions in which theoretical development should go.

It is in this spirit that we present a simple analysis of a hypothetical shelf-circulation that may be supposed to apply to winter-time conditions on the east-coast continental shelf of North America. Winter-time is attractive because ( $i$ ) density is controlled primarily by the salinity distribution, thus limiting sources of buoyancy to river discharge near the coast, and (ii) the weak vertical density stratification in winter permits a simplification in our treatment of the nonlinear salt-transfer equation (1).

\section{Steady semi-infinite shelf model}

Consider a shelf of infinite length ( $y$-direction) and a semiinfinite width (extending from the deep ocean at $x=0$ to negative $x$-infinity). The depth of the shelf is $H$. A mean flux of water, $T_{R}$ per unit length of coast-line, flows toward the sea, due to the cumulative effect of river discharge along the coast. The steady wind-stress components at the surface $z=H$ are $\tau_{s x}$ and $\tau_{s y}$. The density is related to salinity by the simple law $\rho=\rho_{0}(1+\beta s)$. We assume a linear dynamics

$$
\begin{gathered}
\nu \psi_{z z z z}-\beta g s_{x}-f v_{z}=0 \\
\nu v_{z z}+f \psi_{z}=0 \\
\kappa s_{z z}+\psi_{z} s_{x}-\psi_{x} s_{z}=0
\end{gathered}
$$

where the motion is independent of $y, x$-derivatives of diffusive terms are neglected on account of the large ratio of horizontal to vertical scales, and the stream-function $\psi$ defines the component velocities $u=-\psi_{z}$ and $w=\psi_{x}$. The boundary conditions in $z$ are that at $z=0: \psi=\psi_{z}=s_{z}=v=0$; and at $z=H: \psi=-T_{R},-\nu \psi_{z z}=\tau_{s x}, \nu v_{z}=\tau_{s y}, s_{z}=0$.

The flux due to the river discharge is small compared to the fluxes present on the shelf, a fact that we make explicit by introducing the ratio $\epsilon=\left|T_{R} / \Psi\right|$ as a small quantity, where $\Psi$ is the amplitude of the internal circulation on the shelf. We now introduce dimensionless variables in the following way

$$
z=\eta H, x=\xi L_{0}, s=\sigma s_{0}, v=v V, \psi=\phi \Psi
$$

where $L_{0}, V$, and $\Psi$ are still to be determined. The equations are now rewritten

$$
\begin{gathered}
\frac{\nu \Psi}{H^{2}} \phi_{\eta \eta \eta \eta}-\frac{g \beta s_{0}}{L_{0}} \sigma_{\xi}-\frac{f V}{H} \mathrm{v}_{\eta}=0 \\
\frac{\nu V}{H^{2}} \mathrm{v}_{\eta \eta}+\frac{f \Psi}{H} \phi_{\eta}=0 \\
\frac{\kappa}{H^{2}} \sigma_{\eta \eta}+\frac{\Psi}{H L_{0}}\left(\phi_{\eta} \sigma_{\xi}-\phi_{\xi} \sigma_{\eta}\right)=0 .
\end{gathered}
$$

At mid-depths the balance in Eq. 4 may be taken as geostrophic, and the balance of the two terms of Eq. 5 is also assumed, thus yielding the two relations

$$
\frac{g \beta s_{0}}{L_{0}}=\frac{f \dot{V}}{H} ; \quad \frac{\nu V}{H^{2}}=\frac{f \Psi}{H} .
$$

In this way we define the amplitudes

$$
V=\frac{\beta g s_{0}}{f L_{0}} H ; \quad \Psi=\frac{\beta g s_{0}}{f L_{0}} \stackrel{\nu}{f}
$$

The equations thus become

$$
\begin{gathered}
E^{2} \phi_{\eta \eta \eta \eta}-\sigma_{\xi}-\mathrm{v}_{\eta}=0 \\
\mathrm{v}_{\eta \eta}+\phi_{\eta}=0 \\
\sigma_{\eta \eta}+\omega\left(\phi_{\eta} \sigma_{\xi}-\phi_{\xi} \sigma_{\eta}\right)=0
\end{gathered}
$$

where $E=\nu / f H^{2}$ is the Ekman number and $\omega=\left(\beta g s_{0} H /\right.$ $\left.f^{2} L_{0}^{2}\right)(\nu / \kappa)$ is the Lineykin number. The nondimensional boundary conditions are

$$
\begin{gathered}
\text { at } \eta=0: \quad \phi=\phi_{\eta}=\sigma_{\eta}=\mathrm{v}=0 \\
\text { at } \eta=1: \quad \phi_{\eta \eta}=-\frac{1}{E}\left(\frac{f L_{0} \tau_{s x}}{\nu g \beta s_{0}}\right) \equiv-\frac{T_{x}}{E}, \\
\mathrm{v}_{\eta}=\left(\frac{f L_{0} \tau_{s y}}{\nu g \beta s_{0}}\right)=T_{y}, \sigma_{\eta}=0 .
\end{gathered}
$$

Since $\epsilon$ is small, we expand $\phi=\phi_{0}+\epsilon \phi_{1}$. Hence, $\phi_{0}$ vanishes at both $\eta=0$ and $\eta=1$. The function $\phi_{1}$ has the integral $\int_{0}^{1} \phi_{1} d \eta=-1$. 
With observed values of parameter, $\omega$ is small, and hence we expand $\sigma$ as $\sigma=\sigma_{0}+\omega \sigma_{1}+\omega^{2} \sigma_{2}$ to obtain the following ordered set of equations

$$
\begin{gathered}
\sigma_{0 \eta \eta}=0 \\
\sigma_{1 \eta \eta}=-\phi_{0 \eta} \sigma_{0 \xi}+\phi_{0 \xi} \sigma_{0 \eta} \\
\sigma_{2 \eta \eta}=-\phi_{0 \eta} \sigma_{1 \xi}+\phi_{0 \xi} \sigma_{1 \eta}+\frac{\epsilon}{\omega}\left(-\phi_{1 \eta} \sigma_{0 \xi}+\phi_{1 \xi} \sigma_{0 \eta}\right) .
\end{gathered}
$$

The solution of Eq. 10 is a function of $\xi$ only: $\sigma_{0}(\xi)$. Therefore, the second term on the right-hand side of Eq. 11 vanishes and, by integration from $\eta=0$, we find

$$
\sigma_{1 \eta}=-\sigma_{0 \xi} \int_{0}^{\eta} \phi_{0 \eta} d \eta=\phi_{0} \sigma_{0 \xi} \text {. }
$$

Upon integration of Eq. 12 from $\eta=0$ to $\eta=1$, the lefthand side vanishes on account of the boundary conditions and we find the following:

$$
0=\int_{0}^{1}\left(-\phi_{0 \eta} \sigma_{1 \xi}+\phi_{0 \xi} \sigma_{1 \eta}-\frac{\epsilon}{\omega} \phi_{1 \eta} \sigma_{0 \xi}+\frac{\epsilon}{\omega} \phi_{1 \xi} \sigma_{0 \eta}\right) d \eta .
$$

The fourth term vanishes because $\sigma_{0 \eta}=0$. The first term can be integrated by parts to give

$$
-\int_{0}^{1} \phi_{0 \eta} \sigma_{1 \xi} d \eta=-\left.\left(\phi_{0} \sigma_{1 \xi}\right)\right|_{0} ^{1}+\int_{0}^{1} \phi_{0} \sigma_{1 \xi \eta} d \eta
$$

but the first term on the right-hand side vanishes because $\phi_{0}=0$ at $\eta=0,1$. Thus, we have the relation

$$
0=\int_{0}^{1} \phi_{0} \sigma_{1 \xi \eta} d \eta+\int_{0}^{1} \phi_{0 \xi} \sigma_{1 \eta}+\frac{\epsilon}{\omega} \sigma_{0 \xi} .
$$

Inserting the value of $\sigma_{1 \eta}$ from Eq. 13, we obtain the following expression:

$0=-\int_{0}^{1} \phi_{0}\left(\phi_{0} \sigma_{0 \xi \xi}+\phi_{0 \xi} \sigma_{0 \xi}\right) d \eta-\int_{0}^{1} \phi_{0 \xi} \phi_{0} \sigma_{0 \xi} d \eta+\frac{\epsilon}{\omega} \sigma_{0 \xi}$.

The solution of the dynamical problem (Eqs. 7 and 8) is a standard one* of the form

$$
\begin{aligned}
& \text { * } \\
& \phi_{0}^{\prime}=-d_{1}\left[e^{\eta^{\prime}}\left(-\cos \eta^{\prime}+\sin \eta^{\prime}\right)+e^{-\eta^{\prime}}\left(\cos \eta^{\prime}+\sin \eta^{\prime}\right)\right] \\
& -c_{1}\left[e^{\eta^{\prime}}\left(-\cos \eta^{\prime}+3 \sin \eta^{\prime}\right)+e^{-\eta^{\prime}}\left(\cos \eta^{\prime}-\sin \eta^{\prime}\right)\right] \\
& -e^{\eta^{\prime}}\left(\cos \eta^{\prime}-\sin \eta^{\prime}\right)+1 \text {. } \\
& \phi_{0}{ }^{\prime \prime}=d_{2}\left[e^{\eta^{\prime}}\left(-\cos \eta^{\prime}+\sin \eta^{\prime}\right)+e^{-\eta^{\prime}}\left(\cos \eta^{\prime}+\sin \eta^{\prime}\right)\right] \\
& -c_{2}\left[e^{\eta^{\prime}}\left(-\cos \eta^{\prime}+3 \sin \eta^{\prime}\right)+e^{-\eta^{\prime}}\left(\cos \eta^{\prime}-\sin \eta^{\prime}\right)\right] . \\
& \text { where } \eta^{\prime}=\eta / \sqrt{2 E} \text {. } \\
& c_{1}=\frac{1}{\Delta}\left[2 \sin \beta-e^{\beta^{\prime}}\left(\cos \beta^{\prime}+\sin \beta^{\prime}\right)+e^{-\beta^{\prime}}\left(\cos \beta^{\prime}-\sin \beta^{\prime}\right)\right] . \\
& d_{1}=\frac{1}{\Delta}\left[-2 \cos \beta+e^{\beta^{\prime}}\left(3 \cos \beta^{\prime}+\sin \beta^{\prime}\right)\right. \\
& \left.\left.+e^{-\beta^{\prime}\left(\sin \beta^{\prime}\right.}+\cos \beta^{\prime}\right)-2 e^{\beta}\right] . \\
& c_{2}=\frac{1}{\Delta}\left[e^{\beta^{\prime}}\left(-\cos \beta^{\prime}+\sin \beta^{\prime}\right)+e^{-\beta^{\prime}}\left(\cos \beta^{\prime}+\sin \beta^{\prime}\right)\right] . \\
& d_{2}=\frac{1}{\Delta}\left[e^{\beta^{\prime}}\left(-\cos \beta^{\prime}+3 \sin \beta^{\prime}\right)+e^{-\beta^{\prime}}\left(\cos \beta^{\prime}-\sin \beta^{\prime}\right)\right] . \\
& \Delta=-2 e^{\beta}+2 e^{-\beta}+4 \sin \beta, \beta^{\prime}=(2 E)^{-1 / 2}, \beta=2 \beta^{\prime} .
\end{aligned}
$$

$$
\phi_{0}=\left(T_{y}+\sigma_{0 \xi}\right) \phi_{0}{ }^{\prime}(\eta)+T_{x} \phi_{0}{ }^{\prime \prime}(\eta) .
$$

Substituting this solution for $\phi_{0}$ into the above integral, we obtain the following differential equation for $\sigma_{0}$ as a function of $\xi$ :

$$
\begin{aligned}
& {\left[A\left(\sigma_{0 \xi}\right)^{3}+\left(2 A T_{y}+2 B T_{x}\right)\left(\sigma_{0 \xi}\right)^{2}\right.} \\
& \left.\quad+\left(A T_{y}{ }^{2}+2 B T_{x} T_{y}+F T_{x}^{2}\right) \sigma_{0 \xi}-\frac{\epsilon}{\omega} \sigma_{0}\right]_{\xi}=0
\end{aligned}
$$

where the coefficients

$$
A=\int_{0}^{1}\left(\phi_{0}{ }^{\prime}\right)^{2} d \eta, B=\int_{0}^{1}\left({\phi_{0}}{ }^{\prime} \phi_{0}{ }^{\prime \prime}\right) d \eta, F=\int_{0}^{1}\left(\phi_{0}{ }^{\prime \prime}\right)^{2} d \eta
$$

are strong functions of the Ekman number $E$.

Integrating Eq. 14 once, we obtain an expression for the flux of salt in the $\xi$ direction, which must vanish on the shelf, hence the constant of integration also vanishes.

$$
\begin{aligned}
A\left(\sigma_{0 \xi}\right)^{3} & +2\left(A T_{y}+B T_{x}\right)\left(\sigma_{0 \xi}\right)^{2} \\
& +\left(A T_{x}{ }^{2}+2 B T_{x} T_{y}+F T_{y}{ }^{2}\right) \sigma_{0 \xi}-\frac{\epsilon}{\omega} \sigma_{0 \xi}=0 .
\end{aligned}
$$

The width of actual shelves is considerably less than that of the natural scale length $L_{0}$ (as we can see by the fact that at the coast the salinity generally falls to values only slightly less than deep-sea values); hence, we can scarcely expect to fit the functional form of the solution of Eq. 15 to observed salinity distributions. Our main interest, therefore, is in using 15 to estimate $s_{x}$ in the vicinity of $x=0$. Redimensionalizing and setting $s_{x}=s_{0} / L$, we obtain the following cubic for $L$

$$
\begin{aligned}
& L^{3}-\left[\frac{A}{E}\right. \frac{\nu}{T_{R} \kappa} \frac{1}{f^{3} H}\left(\tau_{s y}^{2}+\frac{2 B}{A} \tau_{s x} \tau_{s y}+\frac{F}{A} \tau_{s x^{2}}\right] L^{2} \\
&-2\left[\frac{A}{T_{R}}\left(\frac{\nu}{\kappa}\right) \frac{1}{f}\left(\frac{\beta s_{0} H}{f^{2}}\right)\left(\tau_{s y}+\frac{B}{A} \tau_{s x}\right)\right] L \\
&-\left[\frac{A E}{T_{R}}\left(\frac{\nu}{\kappa}\right) H f\left(\frac{\beta s_{0} H}{f^{2}}\right)^{2}\right]=0 .
\end{aligned}
$$

This equation gives us the natural horizontal scale length $L$ as a function of Ekman number $E$ for various choices of the applied wind stress, river discharge, Coriolis parameter, shelfdepth, and Prandtl number $\nu / \kappa$.

Solutions of $L$ as a function of $E$ are given in Fig. 1. The scale of the ordinate in centimeters is computed on the assumption that the values of the parameters involved are appropriate for the Eastern U.S. Continental shelf: segment 2 (see next section)

$$
\begin{gathered}
f=0.7 \times 10^{-4} \mathrm{sec}^{-1} ; H=5 \times 10^{3} \mathrm{~cm} . \\
\beta g s_{0}=30 \mathrm{~cm} \mathrm{sec}^{-2} ; \quad T_{R}=50 \mathrm{~cm}^{2} \mathrm{sec}^{-1} ; \nu / \kappa=1 .
\end{gathered}
$$

The purely wind-stress driven régimes correspond to the upper portion of the diagram, where the curves have slope -1 . The lower curve, which is convex up, with maximum near $E=3.5 \times 10^{-2}$, is the pure density-driven model with no stresses at the surface. For large Ekman number all curves tend to coalesce, and to be basically density driven. As can be seen, the $y$-stress is much more important in driving 


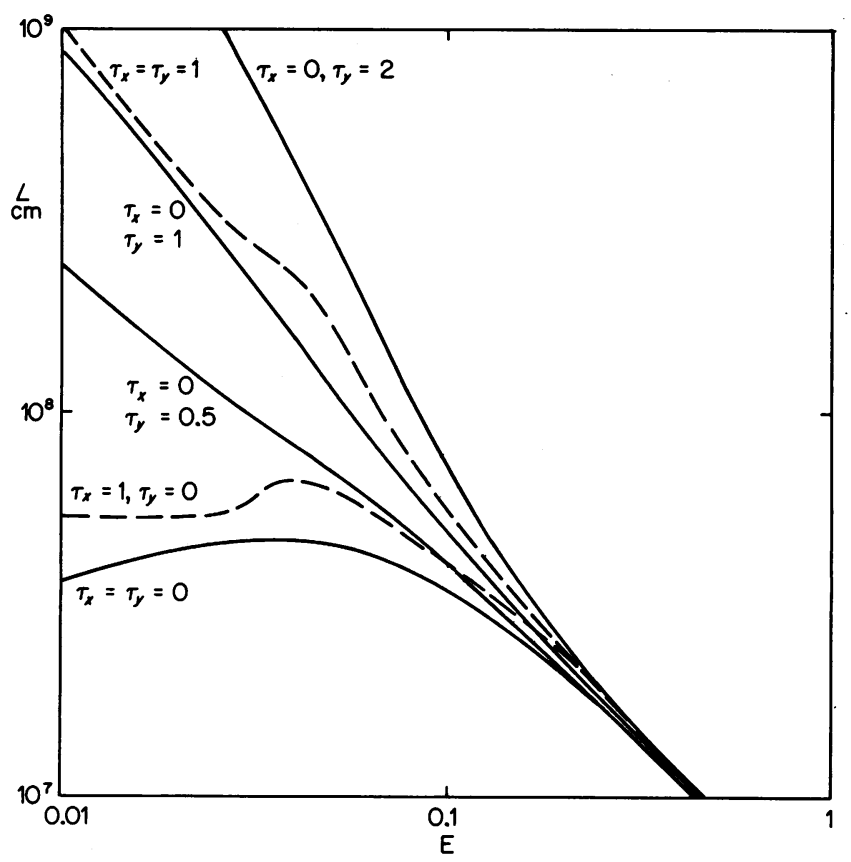

Fig. 1.

than the $x$-stress, which tends to transport water mostly parallel to the isobaths and isohalines.

Physically, for small $E$, with $y$-stress predominant, the opposing Ekman transports that convect salt in the $x$-direction are themselves independent of $E$. The mixing $\kappa$ that "short-circuits" these convective transports is proportional (through unity Prandtl number) to $E$, and since small $E$ corresponds to small shorting, and hence large penetration of salt (large $L$ ), we can understand the inverse relation of $L$ and $E$ in this instance.

When no applied wind-stress acts, the Ekman transports are driven by stress associated with the shear $v_{z}$ produced by the horizontal density gradient. This internal stress diminishes as $E$ becomes very small, and despite a partial compensating effect due to diminishing $\kappa$ the salt penetration indicated by $L$ also diminishes for small $E$, a phenomenon that accounts for the fact that the $L(E)$ form for density alone is different from that for $y$-wind-stress.

On the other hand, for very large $E$ there are no boundary layers, and the exchange of water in the $x$-direction is much inhibited. Vertical mixing is enhanced and, hence, salt penetration is reduced further by the short circuit principle. The case of $y$-stress is most drastically affected because large $E$ is the limit of no rotation, and in that case the $y$-stress no longer drives an $x$-flux of mass. $L(E)$ is therefore powerfully decreased as $E$ gets large. The density effect $s_{0}$ is least affected for large $E$; hence, all curves coalesce with it on an $E^{-1}$ line.

For the whole range of values of $L$ shown in Fig. 1, the quantity $\omega$ is less than 0.1 .

\section{Description of the eastern United States continental shelf}

Bumpus (2) divides the shelf waters off the eastern United States coast into three sections: $(i)$ the Gulf of Maine, (ii) the Middle Atlantic Bight-from Nantucket Shoals to Cape Hatteras, and (iii) the South (sic) Atlantic Bight from Cape Hatteras to Florida. The geometry of $(i)$ is too labyrinthine to lend itself to our model, and the location of (iii) is too perilously close to the Gulf Stream to assert local dynamics. We confine ourselves to a comparison of theory and observation in the region (ii).

The geometry (topography) of the region is well described in Uchupi's (3) chart. Hydrographic and topographic conditions are not altogether uniform along the Middle Atlantic Bight. In order to illustrate the representativeness of values of parameters, we subdivide it into three segments: $(a)$ from Nantucket Shoals to western Long Island, $(b)$ from western Long Island to Cape May, (c) from Cape May to Cape Henry. The length (parallel to the coastline), and the width $\Delta x$ (from the coast to the $200-\mathrm{m}$ isobath) of each of these segments are tabulated in Table 1. The isobaths do not run in the same geographical direction, $\vartheta$, at each segment. Thus, in segment $(a)$ they run nearly east $\left(\vartheta=80^{\circ}\right)$, whereas in (c) they run more nearly northward $\left(\vartheta=25^{\circ}\right)$. The depth $H$ of the shelf varies from zero at the coast to $200 \mathrm{~m}$ (by definition) at the edge of the shelf. The depth does not vary uniformly across the shelf, but there is a middle portion of nearly constant depth. Representative values of this "constant" depth $H$ are also given in Table 1 for each segment. Salinity and temperature on the shelf in February and March is described in various papers (e.g., ref. 4). Although these data are not sufficient for firm statistics, they do indicate a decrease of salinity shoreward, the total drop $\Delta s$ across the shelf being tabulated also in Table 1 . These data also demon-

TABLE 1. Characteristics of the shelf in the Middle Atlantic Bight

\begin{tabular}{|c|c|c|c|c|}
\hline & \multicolumn{3}{|c|}{ Nantucket Shoals-W. Long Is.-Cape May-Cape Henry } & \multirow[t]{2}{*}{ Source } \\
\hline Segment & $(a)$ & (b) & $(c)$ & \\
\hline Length, km & 280 & 180 & 210 & (3) \\
\hline Width, $\mathrm{km} \Delta x$ & 110 & 139 & 96 & (3) \\
\hline Trend, $\vartheta$ & $80^{\circ}$ & $40^{\circ}$ & $25^{\circ}$ & (3) \\
\hline Depth, $H \mathrm{~m}$ & 70 & 50 & 40 & (3) \\
\hline$\Delta s \mathrm{ppm}$ & 1.5 & 1.5 & 2.8 & (4) \\
\hline \multicolumn{5}{|l|}{ River discharge, $R$} \\
\hline $10^{3} \mathrm{ft}^{3} \mathrm{sec}^{-1}$ & 32 & 32 & 98 & (5) \\
\hline$T_{R}, \mathrm{~cm}^{2} \mathrm{sec}^{-1}$ & 33 & 52 & 135 & (5) \\
\hline$\tau_{8 x}$, dynes $\mathrm{cm}^{-2}$ & 0.31 & 1.20 & 1.42 & (6) \\
\hline$\tau_{s y}$, dynes $\mathrm{cm}^{-2}$ & 1.54 & 1.01 & 0.66 & (6) \\
\hline$L, 10^{8} \mathrm{~cm}$ & 2.5 & 3.2 & 1.2 & $s_{0} \Delta x / \Delta s$ \\
\hline
\end{tabular}


strate that the vertical stratification $\delta s$ is very weak, but here the data are insufficient to give reliable means.

Fresh-water discharge due to rivers $R$ in each segment is well documented (5). The quantity $T_{R}$ is the discharge per unit length of shelf in each segment. Winter-time mean windstress over the whole region can be estimated from Hellerman's (6) world charts. The eastward component is 1.57 dynes $\mathrm{cm}^{-2}$ and the northward component is -0.04 dynes $\mathrm{cm}^{-2}$. Because the trend of the isobaths is different from one segment to the other, the values of the offshore stress $\tau_{s x}$ and the longshore component $\tau_{s y}$ are different from one segment to another. The characteristic scale $L$ (defined as $s_{0} \Delta x / \Delta s$ ), which also appears in the theory, is tabulated. Evidently, the real shelf is quite narrow compared to the natural scale of penetration of salt that would occur were the real shelf semi-infinite. It does not seem that this narrowness invalidates the application of the semi-infinite model to the real case, because the net flux of salt is zero in both cases.

Direct measurements of current velocity at $50-\mathrm{m}$ depth and greater in winter are rare, and we can refer to no representative current-meter series. Our main source of information is that deduced from recovery of surface and bottom drifters (2) - which give indications of a general westward surface drift south of New England [segment (a)], and southward in segments $(b)$ and $(c)$. Both of these are in the negative $y$-direction in the model. The amount is quite uncertain-perhaps $5 \mathrm{~cm} \mathrm{sec}^{-1}$.

Our final source of information is mean sea-level along the coast, as given by geodesists - who have determined that sea-level rises from Cape Hatteras to Nantucket shoals by about $20 \mathrm{~cm}$. However, the reality of this rise is now disputed (e.g., ref. 7). The two-dimensional model of preceding section assumes that there is no rise.

\section{Application of the theoretical model to the Middle Atlantic Bight}

In attempting a comparison of the theory and the real Middle Atlantic Bight régime, we do not know a priori how to choose appropriate values of $\nu$ and $\kappa$. We may assume that $\nu / \kappa=$ 1. Entering Fig. 1 with observed scale-length $3.2 \times 10^{8} \mathrm{~cm}$, and with stresses $\tau_{s x}=1, \tau_{s y}=1$, we find an Ekman number of $2.6 \times 10^{-2}$; this value corresponds to a boundary layer thickness of $7.5 \mathrm{~m}$. The point on Fig. 1 corresponding to these values lies well away from the curve for density control alone; hence, the indication is that the shelf régime is essentially wind-driven. The vertical stratification $\delta s$ may be computed from the expression $\delta s=s_{0} T_{R} f / \tau_{s y}$, and is found to be approximately $4 \times 10^{-3} s_{0}$, or $0.14 \mathrm{ppm}$. This seems to be of the correct order of magnitude, but a long time-series of winter-time current and density measurements is required to obtain an observational verification of the amplitude of the mean current field and of the mean vertical structure in current and density. A serious discrepancy exists between the predicted v-component of velocity, and the observed value. All observations suggest a negative $v$-velocity of the order of $5 \mathrm{~cm} \mathrm{sec}^{-1}$. The theoretical $v$-component is positive, about $20 \mathrm{~cm} \mathrm{sec.}^{-1}$ The positive theoretical geostrophic velocity is necessary to give a bottom Ekman layer with transport that matches that of the surface layer.

The discrepancy can be easily resolved if the reality of the observed rise of sea-level along the coast is admitted. If we denote the surface of the sea by $h$, then the slope in sea-level (sea-level is not level) causes a $y$-component of pressure gradient that appears to be nearly in balance with the applied surface wind-stress at depths of $50 \mathrm{~m}$ :

$$
\tau_{y s} \cong g H \frac{\partial h}{\partial y}
$$

In this case, the return flow shoreward, which compensates for the Ekman transport offshore in the surface layer, does not need to occur as a bottom Ekman layer, but occurs at all depths as a broadly distributed shoreward geostrophic flow. The form of $u_{0}(z)$ is different from the case of the second section, but the amplitude of the transport is the same; hence, we anticipate that the resulting Ekman number will be much the same as before, and we can calculate it neglecting the stress due to the geostrophic shear. The velocity fields can be written as sums of geostrophic and Ekman parts (denoted by subscripts $g$ and $e$ )

$$
\begin{gathered}
u_{0}=u_{o}+u_{e}=-\frac{\tau_{y s}}{f H}+u_{e} \\
v_{0}=v_{o}+v_{e}=-\frac{g \beta}{f} \frac{\partial s_{0}}{\partial x}\left(z-\frac{H}{2}\right)+\frac{\tau_{x s}}{f H}+v_{e}
\end{gathered}
$$

where $u_{e}$ and $v_{e}$ are functions that differ from zero only in a region close to $z=H$, and whose vertical integrals are

$$
\int_{0}^{H} u_{e} d z=\frac{\tau_{y s}}{f} \quad \int_{0}^{H} v_{e} d z=-\frac{\tau_{x s}}{f}
$$

and where we have assumed that the wind set-up entirely balances the $y$-stress at the surface, and where we have assumed that the barriers at Cape Hatteras and Nantucket Shoals completely block the flow at 50-m depth, so that not only do we assert $\int_{0}^{H} u_{0} d z=0$, but also $\int_{0}^{H} v_{0} d z=0$. The effective horizontal diffusivity in the $\mathrm{x}$-direction is essentially

$$
A_{x}=\left(\tau_{y s} / f\right)^{2} / 3 \kappa
$$

(one-third the value for the model with two Ekman layers) and, hence, the horizontal scale length is simply

$$
L=A_{x} H / T_{R}
$$

Solving for $\kappa$, and using the value of parameters in segment 2 of the Middle Atlantic Bight, we obtain

$$
\kappa=30 \mathrm{~cm} \mathrm{sec}^{-1}
$$

with $\nu / \kappa=1$, this corresponds to an Ekman number $1.7 \times$ $10^{-2}$, and layer depth $6.5 \mathrm{~m}$. The average velocity components in the Ekman layer are thus $u=2.2, v=-1.9 \mathrm{~cm} \mathrm{sec}^{-1}$, at $z=H / 2, u=0, v=0.3 \mathrm{~cm} \mathrm{sec}^{-1}$, and at $z=0, u=0, v=$ $0.6 \mathrm{~cm} \mathrm{sec}^{-1}$. These values are more consistent with the observed drift. It would seem, therefore, that verification of the slope of sea-level along the coast is of crucial importance for understanding the dynamics of the shelf waters.

There remains the question of the effect of time-varying currents. Inspection of daily wind records at Nantucket Shoals Light vessel shows that the winter-time root-meansquare wind-stress exceeds the mean by a factor of 5-10. Thus, transient fluxes in the Ekman layer exceed the mean by about the same amount. The effective horizontal eddy diffusivity is proportional to the square of these fluxes during the intervals when the storms are blowing. This may very well affect the relationships shown in Fig. 1. However, the complexities introduced with time variability are too great 
to pursue theoretically without some guidance from observational time-series of current and density as functions of depth. For example, what is the vertical modal structure of horizontal displacements of water due to a typical winter storm? How is the intensity of mixing within the watercolumn related to such a storm?

As information becomes available, it will also be interesting to find the position of points on the $E-L$ diagram for other shelves.

\section{Pollution distributions}

Once the particular régime that applies to a shelf has been determined, it is possible to make calculations of the distribution of a tracer or pollutant introduced, like river water, at the coast, or at a point source, or in an initially concentrated patch that spreads with time. Conversely, properly conducted tracer experiments can, in principle, help verify the identification of which mechanism is acting on a given shelf.

For example the effective eddy coefficients in three directions for the previous example, with sea-level slope, can be shown to be

$$
\begin{aligned}
& A_{x}=\left(\tau_{y s} / f\right)^{2} / 3 \kappa \\
& A_{y}=\left[\left(\tau_{x s} / f\right)^{2} / 3 \kappa\right]+\left[\frac{1}{120 \kappa}\left(\frac{g \beta s_{0} H}{f}\right)^{2}\left(\frac{H}{L}\right)^{2}\right] \\
& A_{z}=\kappa
\end{aligned}
$$

For the Middle Atlantic Bight, the orders of magnitude of these coefficients turns out to be

$$
\begin{aligned}
& A_{x}=2.28 \times 10^{6} \mathrm{~cm}^{2} / \mathrm{sec} \\
& A_{y}=2.61 \times 10^{6} \mathrm{~cm}^{2} / \mathrm{sec}
\end{aligned}
$$

$$
A_{z}=30 \mathrm{~cm}^{2} / \mathrm{sec}
$$

Thus, whatever the original vertical distribution, there will be near-vertical homogeneity within three days. Then, horizontal diffusion will spread the pollutant in a growing area of roughly circular shape, with $x$-diameter $140 \mathrm{~km}$ and $y$ diameter $150 \mathrm{~km}$ after about 100 days, at which time the patch occupies the full width of the shelf. The motion of the center of the ellipse is quite slow.

The time for this lateral dispersion of the tracer over the width of the shelf is interesting because it shows us that the steady-state régime could be established in a time less than one winter-time season and, hence, has sufficient time to approach a steady state.

Support for this study came from the National Science Foundation-Grant GA-30729X. Helpful comments and discussion were offered by Drs. George Veronis, Dennis Moore, Robert Beardsley, William Simmons, and Mr. Kuh Kim.

1. Taylor, G. I. (1953) "Dispersion of soluble matter in a solvent flowing slowly through a tube," Proc. Roy. Soc. Ser. $A$ 219, 446-468.

2. Bumpus, D. F. (1972) "A description of the circulation on the continental shelf of the east coast of the United States," Progr. Oceanogr., in press.

3. Uchupi, E. (1965) Map showing relation of land and submarine topography, Nova Scotia to Florida, U.S. Geol. Surv. Misc. Geol. Invest. Map 1-451.

4. Bigelow, H. B. \& Sears, M. (1935) Studies of the water on the Continental Shelf, Cape Cod to Chesapeake Bay II Salinity PPOM IV , 1, 94pp.

5. Bue, C. D. (1970) U.S. Geol. Surv. Water-Supply Pap., 1899-I.

6. Hellermann, S. (1967) "An updated estimate of the windstress on the world ocean," Mon. Weather Rev. 95, 607-626.

7. Montgomery, R. B. (1969) "Comments on oceanic leveling," Deep-Sea Res. 16, 147-152. 